\title{
Smart Interpolation by Anisotropic Diffusion
}

\author{
S. Battiato, G. Gallo, F. Stanco \\ Dipartimento di Matematica e Informatica \\ Viale A. Doria, 6-95125 Catania \\ \{battiato,gallo,fstanco\}@dmi.unict.it
}

\begin{abstract}
To be able to enlarge a digital image from a single frame preserving the perceptive cues is a relevant research issue. The best algorithms take into account the presence of edges due to the variation of luminance, to interpolate correctly the original samples/pixels of the original image. This produces pictures where the interpolated artifacts (aliasing blurring effect, ...) are limited. The zooming algorithm proposed in this paper reduces the noise and enhance the contrast to the borders/edges of the enlarged picture using classical anisotropic diffusion improved by a smart heuristics strategy. The method requires limited computational resources and it works on graylevel images, RGB color pictures and Bayer data. Our experiments show that this algorithm outperforms in quality and efficiency the classical interpolation methods (replication, bilinear, bicubic).
\end{abstract}

\section{Introduction}

The problem of producing an enlarged picture from a given digital image is a relevant research issue. This problem arises frequently whenever a user wishes to zoom in to get a better view of a given picture. As known, there are several issues to take into account about zooming: unavoidable smoothing effects, reconstruction of high frequency details without the introduction of artifacts and computational efficiency. Several good zooming techniques are nowadays well known [9], [10], [13], [16], [18], [22].

A zooming algorithm provides as output a picture that has greater size than the input image preserving as much as possible the information content of the original image. The commercial digital image processing software usually interpolate the original samples to obtain the missing information; pixel replication, bilinear or bicubic interpolation are the most popular choices. Unfortunately, these techniques give images whose smoothing effects are evident.
The research of new heuristic/strategies able to outperform classical image processing techniques is nowadays the key-point to produce digital consumer engine (e.g. Digital Still Camera, 3G Mobile Phone, etc.) with advanced imaging application [4].

The research is oriented to obtain good images starting from two or more frame of the same scene (high dynamic range [1], super resolution [5]). This technique gives good images, but needs greater computational complexity. For these reasons the zooming methods remains the most diffused in commercial software.

The Locally Adaptive Zooming Algorithm (LAZA) proposed in [3] takes into account local discontinuities or sharp luminance variations to perform a gradientcontrolled, weighted interpolation. The method speeds up the entire adaptive process without requiring a preliminary gradient computation because the relevant information is collected during the zooming process. Although not linear, it is simple and it could hence be implemented with limited computational resources. The performance of this algorithm in term of PSNR, on the other hand, is far from users requirement.

The new algorithm proposed in this paper (Smart Interpolation by Anisotropic Diffusion - SIAD) first performs the anisotropic diffusion over an image obtained from the original with a large interpolation. This assures that the "flat" areas produced by the anisotropic diffusion will have limited sizes. The image is then obtained by properly subsampling the enlarged image. Our experiments show that the proposed method outperforms in quality and efficiency the classical interpolation methods (replication, bilinear, bicubic) and the LAZA algorithm.

The rest of the paper is organized as follows. Section 1 briefly describes some details of the anisotropic diffusion algorithm adopted. Section 2 provides a detailed description of the proposed algorithm. Section 3 reports the experimental results obtained working on a large dataset of input images. Section 4 shows a possible extension of the proposed strategy. Section 5 concludes the paper. 


\section{Anisotropic diffusion}

To enhance the resolution of a digital image the edges/details must be preserved in a proper way. The first diffusion equation to reduce noise and enhance contrast in region that correspond to borders between different objects within an image, was introduced by Koenderink [15] and Hummel [11]. They suggest the creation of piecewise constant images with step discontinuities at region boundaries.

A non-linear version of this equation was introduced by Perona and Malik [20]. In such formulation, image intensity is treated as a conserved quantity and allowed to diffuse over time, with the amount of diffusion at a point being inversely related to the magnitude of the intensity gradient at that location. This process produces visually impressive results in terms of the creation of sharp boundaries separating uniform regions within an image, but is computationally expensive.

An alternative approach to nonlinear image enhancement was developed by Nizberg and Shiota [19], whose nonlinear filter has excellent performance, comparable to nonlinear diffusion methods; they also introduce some theoretical basis including the notion of "offset filtering". Specifically, Nizberg and Shiota introduced an offset term which displaces kernel enters away from presumed edge locations, thus enhancing the contrast between adjacent regions without blurring their boundary. This application requires complex kernels and is therefore still slow.

Fischl and Schwartz [8] separate the estimation of an offset vector field from image filtering per se, to obtain a simpler, more robust and faster class of algorithms. This allows the application of the desired filter to the original image, and then it uses the offset vector field to produce the final result by a simple pixel permutation. Offset filtering requires the generation of a vector field over the image domain, which specifies an appropriate displacement at each point. The offset pushes the filter into an interior of a region, to be more representative and it doesn't use pixels of different regions.

Leu [17] suggests enhancing of an image reducing edge's width by edge sharpening. There are two major steps in this approach. In the first step, three intensity indices and three gradient indices for each pixel are obtained. In the second step such indices are used to determine how the intensity of the pixel should be adjusted. As suggested by authors such technique can be easily adapted to obtain an efficient anisotropic diffusion. In our experiments we properly set the corresponding parameters to use the last technique as anisotropic diffusion algorithm.

\section{Algorithm description}

In this section we give a detailed description of the proposed algorithm (SIAD). If $L$ is the low level image of size $M x N$, the algorithm proceeds following these steps:

Step 1. The image $L$ is enlarged using classical interpolation methods (e.g. bicubic). The new image $B$, whose size is $K M x K N,(K=8$, in all our experiments $)$ is obviously strongly smoothed due the large interpolation.

Step 2. To reconstruct the edge in $B$ and hence its high frequency content, an algorithm of anisotropic diffusion is performed. This method reduces noise and enhances contrast in regions that correspond to borders between different objects. The new image $A$ appears with evident homogeneous regions while the discontinuities between region boundaries are increased and "enriched" with high frequencies. On the other side, unfortunately, some of them just introduce aliasing artifacts.

Step 3. In order to reduce the aliasing over the image $A$, a suitable low pass filter is hence applied. As results many artifacts guessed in the previous step will be discarded. The smoothed images $A^{\prime}$ is reduced using a weighted averaging technique to obtain $H$ whose dimensions are now $2 M x 2 N$.

In some sense the large interpolation coupled with the anisotropic diffusion allows to isolate the high-frequency component needed to build a zoomed version of the input image.

Crucial to the quality of the final zoomed pictures is the fine tuning of the parameters controlling the anisotropic diffusion.

\section{Experimental results}

The validation of a zooming algorithm requires the assessment of the visual quality of the zoomed pictures. Figure 1 shows some results obtained with the proposed method applied on two gray level images. Unfortunately this qualitative judgement is strictly subjective. For this reason we have used the classical PSNR measure between the original picture and the reconstructed picture to assess the quality of reconstruction. The input image are first subsampled by simple decimation and then interpolated by a factor 2 using the SIAD method.

In particular the algorithm has been tested on 100 gray scale pictures at different resolution levels and different categories (graphics and natural scenes). The images have been acquired through a digital still camera, using a scanner or from clipart CDs.

For sake of comparison we have used simple replication, bicubic interpolation, and LAZA [3] as three comparing stones to assess the quality of our technique. 


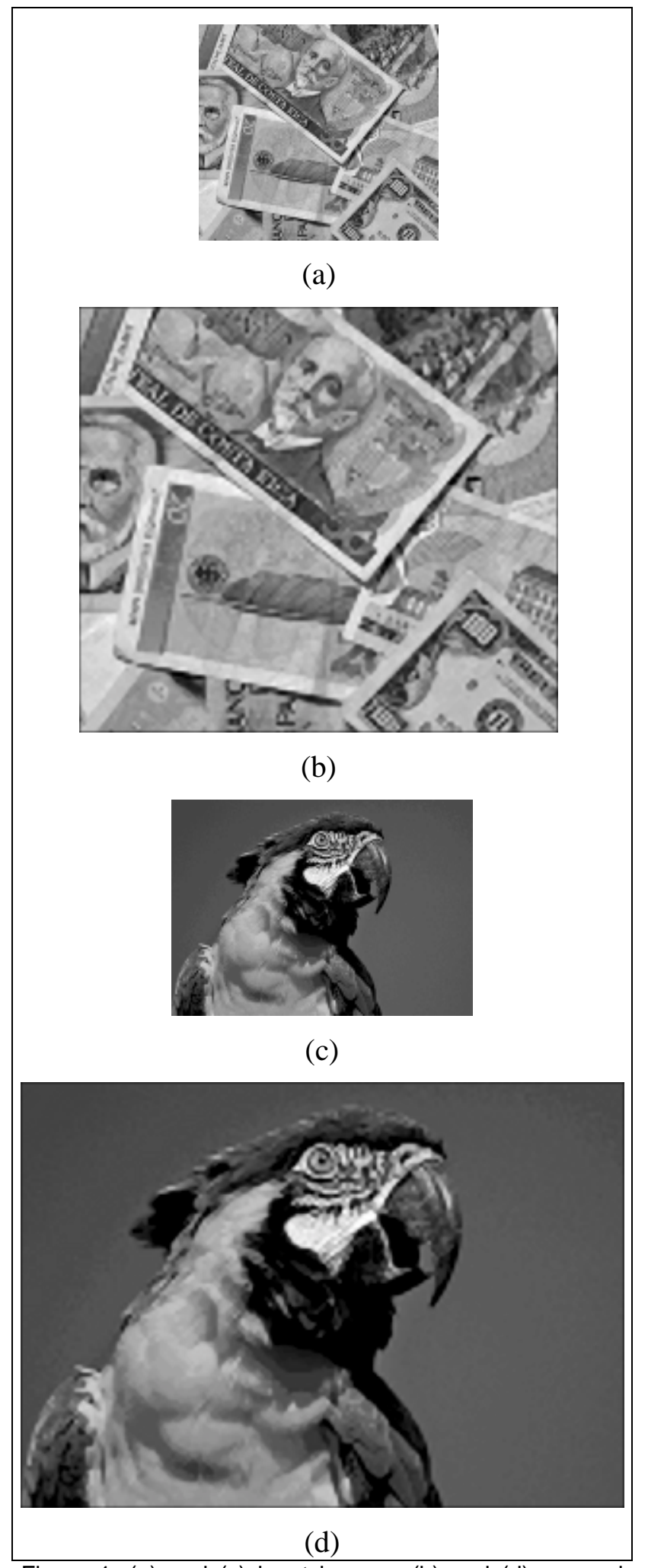

Figure 1. (a) and (c) input images; (b) and (d) zoomed images by SIAD algorithm

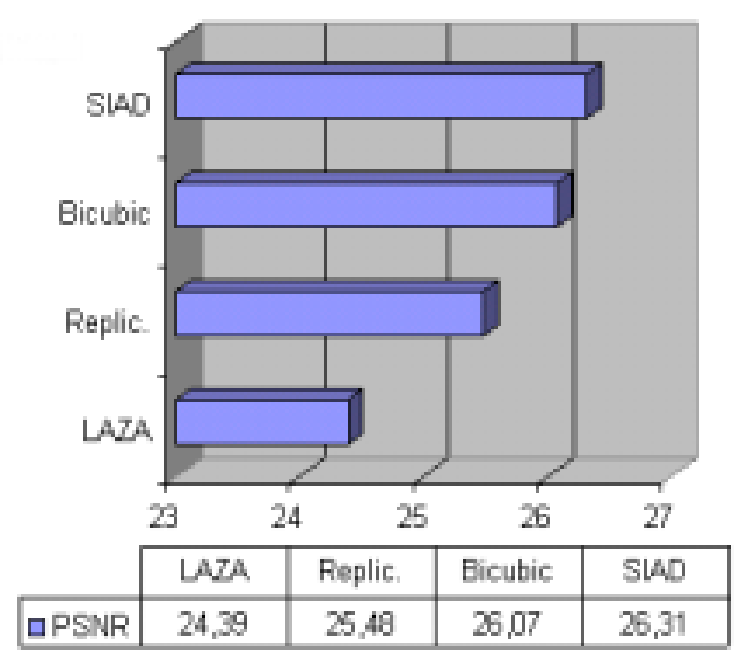

Table I. Averaged PSNR value in Db measured over a test pools of digital images.

In table I are reported the average PSNR values obtained with different algorithms.

The SIAD algorithm always gives better results than the others techniques. This is strictly related with the high frequencies properly guessed by the anisotropic process. Also by a visual inspection the proposed method show performance comparable with both classical interpolation methods and some edge adaptive techniques. Figure 2 shows the same image zoomed by different techniques. It is possible to see how sharpness in the zoomed SIAD image is sensibly increased. The algorithm can be used, of course, with different zooming factors but the corresponding parameters (i.e. the large interpolation, the anisotropic powerness, ...) have to be properly tuned.

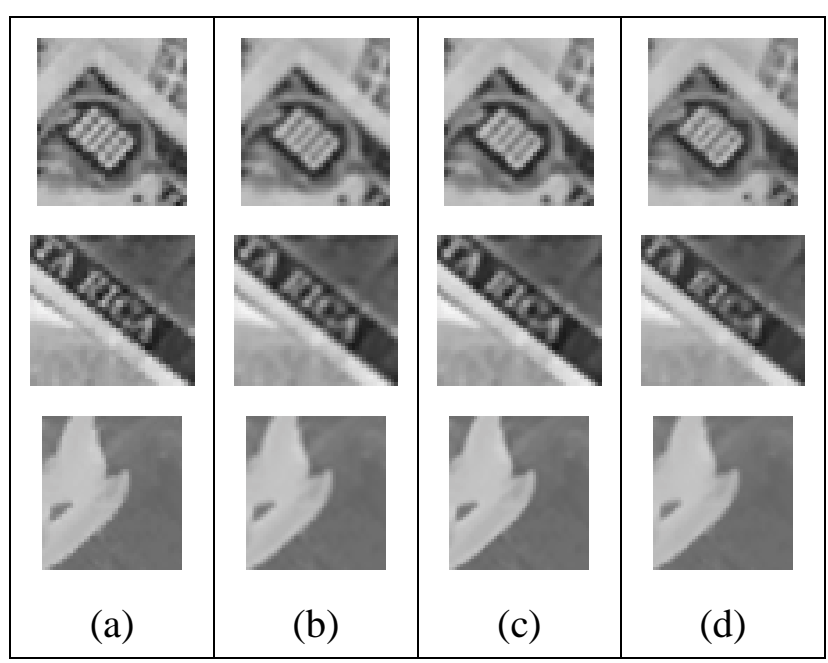

Figure 2. (a) pixel replication; (b) Bicubic interpolation; (c) SIAD; (d) LAZA. 


\section{Some application}

\subsection{Super resolution}

The zooming algorithm is the process to enlarge a picture preserving the details. If we have two or more frame of the same scene, it possible to obtain a sharpen image, using the super resolution techniques [7]. Usually, some Digital Cameras allow choosing the best frame between the numerous frames acquired simultaneously (e.g. bracketing). With the super resolution it is possible to merge such Low-Resolution (LR) frames to obtain a new enhanced picture, where the relative misalignment between successive frames of the same scene allows to recover more high frequency details. In this way the physical limits of the acquisition system are substantially bypassed by properly using more than one frame.

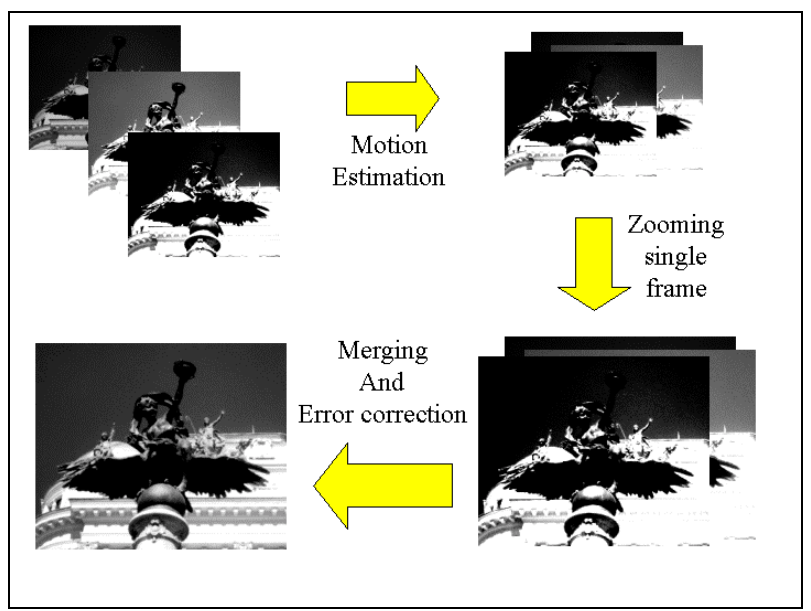

Figure 3. The super resolution scheme.

The first step of the super resolution process is the alignment of the frame [14]. After this step the frame are zoomed using some well-known technique, and then the information relative to the corresponding pixels are merged in some way (e.g. by averaging). See Figure 3 for a schematic description of such process. The resulting image is adjusted by an iterative error correction ([5], [12]). In particular we have zoomed the single frame by replication, bicubic, LAZA [3] and SIAD. This process allows to sensibly improve the visual quality of the image, as reported by our experiments. Table II reports the average PSNR values of the super resolution algorithm applied on some LR-sequences of an image. The SIAD gives the best results both in visual quality and numerically.

Some visual SIAD super resolution results are reported in Figure 4. A detailed description about such comparisons can be found in [2].

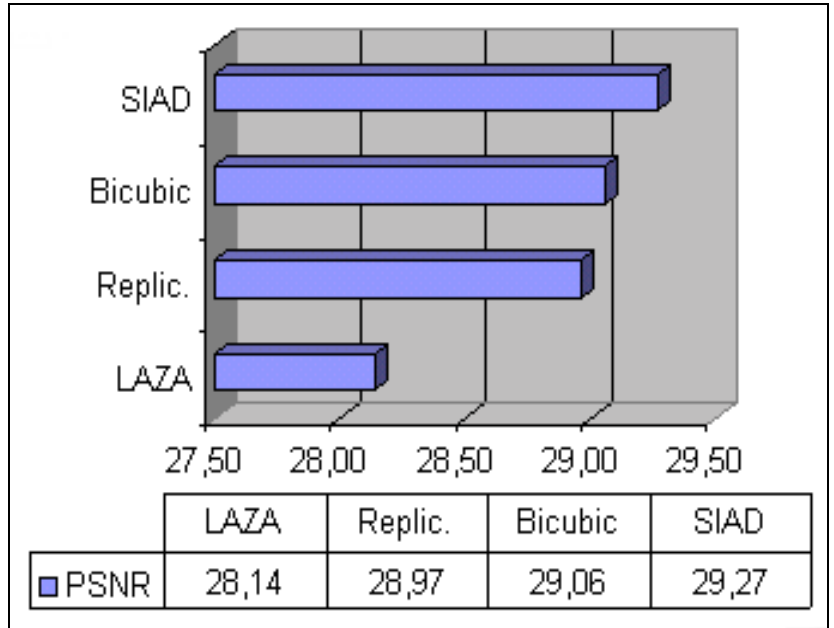

Table II. Averaged PSNR value in Db measured over a test pool of digital images in case of super resolution.

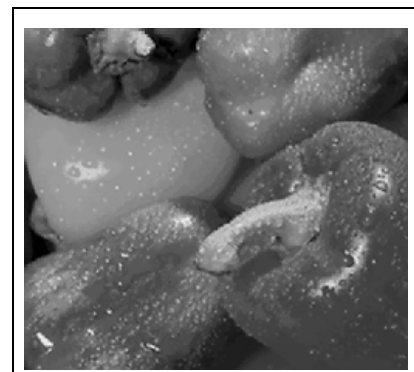

(a)

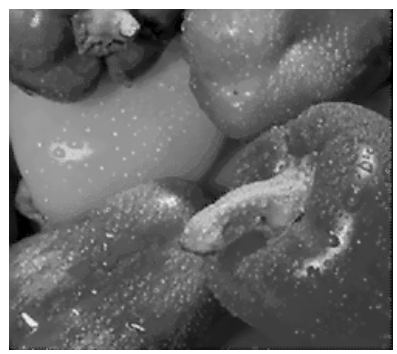

(c)

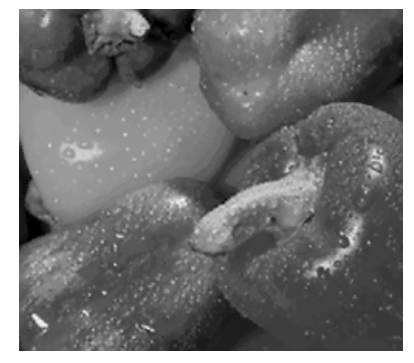

(b)

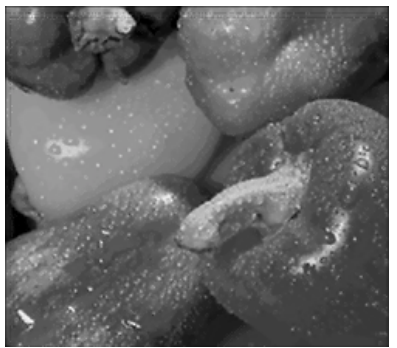

(d)
Figure 4. Super resolution by (a) replication; (b) bicubic interpolation; (c) LAZA; (d) SIAD.

\subsection{Working on Bayer data}

The method has been generalized to work with Bayer data images [6], acquired by CCD/CMOS sensor in almost all digital camcorder. Each pixel, using suitable CFA (Color Filtering Array) array, preserves the intensity of just one of the many color separations. Working in the Bayer domain requires a little effort to readapt ideas and techniques to the new particular environment but allows to improve significantly the quality of the final image reducing at the same time the associated computational complexity. 
In order to preserve the details of the original images, without introducing visible artifacts, the input nxn Bayer image is split into three sub-planes, R, G, B obtained retaining the corresponding chromatic component having, respectively, $n / 2 x n / 2, n x n / 2$ and $n / 2 x n / 2$ pixels. The proposed zooming algorithm is then applied, independently, for each one of these color planes. Combining together these intermediate results a new zoomed Bayer data images is obtained. The image obtained is not an RGB image because the proposed algorithm is not applied as a color reconstruction algorithm. The image is successively interpolated with a simple color interpolation algorithm [21]. Figure 5 shows two related example.

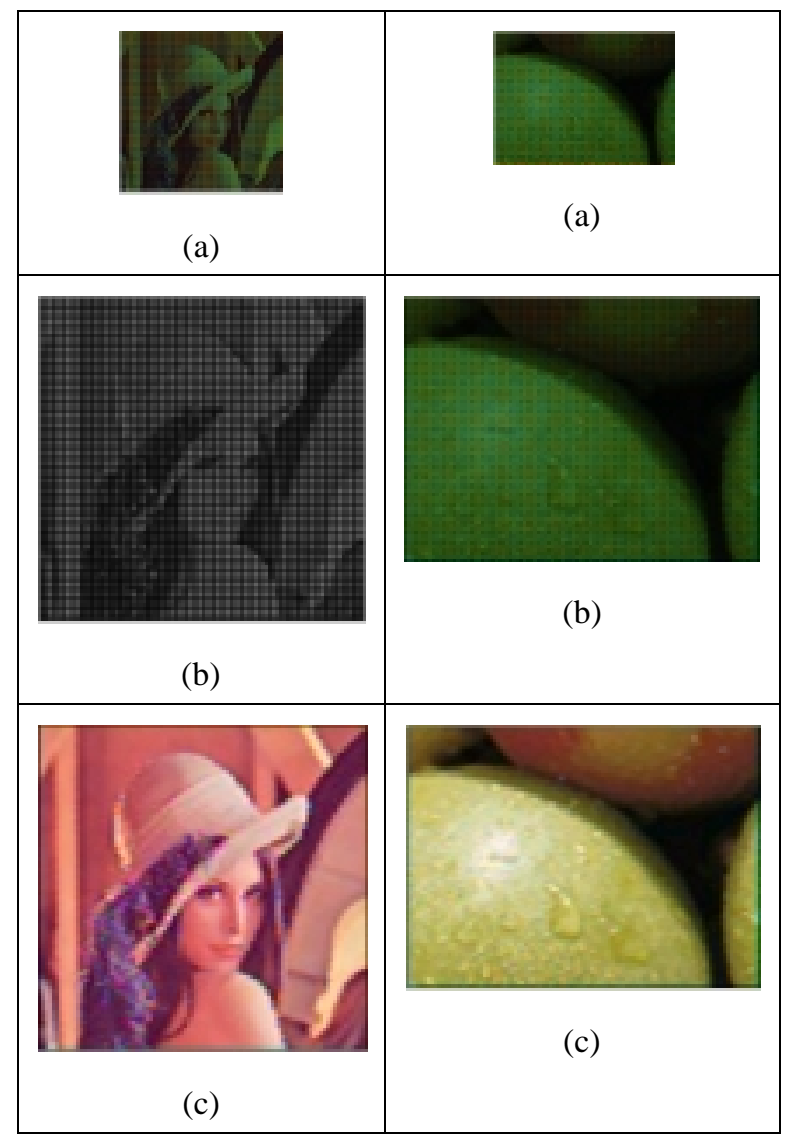

Figure 5. (a) Original Bayer pattern; (b) zoomed Bayer pattern using SIAD; (c) RGB zoomed image;

We claim that working directly in the Bayer domain, before color interpolation algorithm, is possible to improve furtherly the quality of the final zoomed image. Further experiments must be done in order to be able to manipulate such images in a more proper way.

\section{Conclusions}

In this paper we have proposed a new technique for zooming a digital picture. The proposed method has been compared to bicubic interpolation, pixel replication and LAZA. The experimental results show that the proposed method provides better qualitative and numerical results.

\section{References}

[1] Battiato S., Castorina A., Mancuso M., High Dynamic Range Imaging: Overview and Application, Accepted for publication: SPIE Journal of Electronic Imaging, 2003;

[2] Battiato S., Gallo G., Mancuso M., Messina G., Stanco F., Analysis and Characterization of Super-Resolution Reconstruction Methods - In proceedings of SPIE Electronic Imaging 2003 - Sensors, Cameras, and Applications for Digital Photography Vol. 5017B-37, Santa Clara, CA USA, 2003;

[3] Battiato S., Gallo G., Stanco F., A Locally Adaptive Zooming Algorithm for Digital Images - Elsevier Image and Vision Computing, 20/11 pp. 805-812, 2002;

[4] Battiato S., Mancuso M., An Introduction to the Digital Still Camera Technology - ST journal of System Research, Special Issue on Image Processing for Digital Still Camera 2 (2), 2001;

[5] Battiato S., Mancuso M., Messina G., Buemi A., Improving Image Resolution by Adaptive Back-Projection Correction Techniques - IEEE Transaction on Consummer Electronics, Vol. 48, issue 3, pp. 409-416, 2002;

[6] Bayer B.E., Color Imaging Array - US Patent 3,971,065 - 1975;

[7] Chaudhuri S., Super-Resolution Imaging - Kluwer Academic Publishers 2001;

[8] Fischl B., Schwartz E.L., Adaptive Nonlocal Filtering: a Fast Alternative to Anisotropic Diffusion for Image Enhancement - IEEE Transactions on PAMI, vol. 21, n. 1, January 1999;

[9] Florencio D.F., Scafer R.W., Post-sampling Aliasing Control for Images - Proceedings of international Conference on Acoustics, Speech and Signal Processing, Detroit, MI 2, pp. 893-896, 1995;

[10] Hou H.S., Andrews H.C., Cubic Splines for Image Interpolation and Digital Filtering - IEEE Transactions on Acoustics, Speech, Signal Processing ASSP-26 (6), pp. 508517, 1978;

[11] Hummel A., Representations Based on Zero-Crossing in Scale-Space - M. Fichler, and O. Firschein, eds., Reading in Computer Vision: Issues, Problems, Principles and Paradigms. Los Angeles, CA; M. Kaufmann, 1986;

[12] Irani M., Peleg S., Super Resolution From Images Sequences - IEEE Conference of Pattern Recognition, vol. 18, pp. 115-120, 1990

[13] Keys R.G., Cubic Convolution Interpolation for Digital Image Proceeding - IEEE Transactions on Acoustics, Speech, Signal Processing 29 (6), pp. 1153-1160, 1981; 
[14] Kim W., Koo Y., An Image Enhancing technique Using Adaptive Sub-Pixel Interpolation for Digital Still Camera System - IEEE transaction on Consummer Electronics, vol. 45, n. 1, February 1999

[15] Koenderink J., The Structure of Image - Biological Cybernetics, vol. 50, pp. 363-370, 1984;

[16] Lee S.W., Palik J.K., Image Interpolation Using Adaptive Fast B-spline Filtering - Proceedings of International Conference on Acoustics, Speech, and Signal Processing. Vol. 5, pp. 177-179, 1993;

[17] Leu J.G., Edge Sharpening through Ramp Width Reduction - ELSEVIER Image and Vision Computing 18 (2000), 501-514;

[18] Monro D.M., Wakefield P.D., Zooming with Implicit Fractals - Proceedings of International Conference on Image Processing ICIP)/ 1, pp. 913-916, 1997;

[19] Nitzberg M., Shiota T., Nonlinear Image Filtering with Edge and Corner Enhancement - IEEE Transactions on PAMI, vol. 14, n. 8, August 1992;

[20] Perona P., Malik J., Scale-Space and Edge Detection Using Anisotropic Diffusion - IEEE Transactions on PAMI, vol. 12, n. 7, July 1990;

[21] Sakamoto T., Nakanishi C., Hase T., Software Pixel Interpolation for Digital Still Cameras Suitable for a 32-bit $M C U$ - IEEE Transaction on Consumer Electronics 44 (4), pp. 1342-1352, 1998;

[22] Sankar P.V., Ferrari L.A., Simple Algorithms and Architecture for B-spline Interpolation - IEEE Transaction on Pattern Analysis Machine Intelligence PAMI-10, pp. 271276, 1998; 